\title{
Correction to: Structure-guided engineering of a Thermobifida fusca cutinase for enhanced hydrolysis on natural polyester substrate
}

Qilei Dong ${ }^{1,3+}$, Shuguang Yuan ${ }^{4 \dagger}$, Lian $\mathrm{Wu}^{1+}$, Lingqia Su${ }^{5}$, Qiaoling Zhao ${ }^{1}$, Jing Wu ${ }^{5}$, Weixue Huang ${ }^{{ }^{*}}$ and Jiahai Zhou ${ }^{1,2^{*}}$ (1)

\section{Correction to: Bioresour. Bioprocess. (2020) 7:37 https://doi.org/10.1186/s40643-020-00324 $-8$}

In the originally published version of the article, a reference and its citation was missed out. The reference citation is included in a new sentence under the heading "Results and discussion".

The corrected first three sentences of the results and discussion section should read as below:

\section{Results and discussion}

Complex structure of $T$. fusca cutinase with cutin mimic Seeking to elucidate how T. fusca cutinase binds to cutin, we collected the diffraction data set and determined the $1.54 \AA$ apo-form crystal structure of T. fusca cutinase (Additional file 1: Fig. S1, S2a, Table S1) in 2010 via molecular replacement using the atomic coordinates of Streptomyces exfoliates lipase (PDB ID: 1JFR) as a searching model (Wei et al. 1998). In 2014, the crystal structures of T. fusca cutinase variant, including the apo and complex with inhibitor (PDB ID 4CG1, 4CG2 and 4CG3), were reported (Roth et al. 2014). In 2017, the

The original article can be found online at https://doi.org/10.1186/s4064 3-020-00324-8

\section{*Correspondence: wxhuang@sioc.ac.cn; jiahai@sioc.ac.cn}

${ }^{\dagger}$ Qilei Dong, Shuguang Yuan and Lian Wu contributed equally to this work

${ }^{1}$ State Key Laboratory of Bioorganic and Natural Products Chemistry, Center for Excellence in Molecular Synthesis, Shanghai Institute of Organic Chemistry, Chinese Academy of Sciences, 345 Lingling Road, Shanghai 200032, China

Full list of author information is available at the end of the article structure of cutinase 1 from Thermobifida cellulosilytica (PDB ID 5LUI) which showed 99\% sequence identity with T. fusca cutinase was published (Dong et al. 2020). Structure superposition showed that the two structures are almost same with RMSD of 0.165 except for the C-terminal loop region (residues 245-253) (Additional file 1: Fig. S3) (Doris Ribitsch 2017).

\footnotetext{
Author details

${ }^{1}$ State Key Laboratory of Bioorganic and Natural Products Chemistry, Center for Excellence in Molecular Synthesis, Shanghai Institute of Organic Chemistry, Chinese Academy of Sciences, 345 Lingling Road, Shanghai 200032, China. ${ }^{2}$ Shaanxi Key Laboratory of Natural Products \& Chemical Biology, College of Chemistry and Pharmacy, Northwest A\&F University, 3 Taicheng Road, Yangling 712100, Shaanxi, China. ${ }^{3}$ State Key Laboratory of Medical Chemical Biology, Nankai University, Tianjin 300071, China. ${ }^{4}$ Shenzhen Institutes of Advanced Technology, Chinese Academy of Sciences, Shenzhen 518055, China. ${ }^{5}$ State Key Laboratory of Food Science and Technology, Jiangnan University, 1800 Lihu Avenue, Wuxi 214122, China.
}

Published online: 27 August 2020

\section{References \\ Dong Q, Yuan S, Wu L, Su L, Zhao Q, Wu J, Huang W, Zhou J (2020) Structure- guided engineering of a Thermobifida fusca cutinase for enhanced hydrolysis on natural polyester substrate. Bioresour Bioprocess. 7:37. https ://doi.org/10.1186/s40643-020-00324-8 \\ Roth C, Wei R, Oeser T, Then J, Follner C, Zimmermann W, Strater N (2014) Structural and functional studies on a thermostable polyethylene tere- phthalate degrading hydrolase from Thermobifida fusca. Appl Microbiol Biotechnol 98(18):7815-7823}

\section{Publisher's Note}

Springer Nature remains neutral with regard to jurisdictional claims in published maps and institutional affiliations. 